\title{
Statistical inference for the shape parameter change-point estimator in negative associated gamma distribution
}

\author{
Chang-chun Tan ${ }^{1 *}$, Bai-qi Miao ${ }^{2}$ and Xing-cai Zhou ${ }^{3}$
}

\section{"Correspondence:}

cctan@ustc.edu.cn

${ }^{1}$ School of Mathematics, Hefei

University of Technology, Heifei,

Anhui 230009, China

Full list of author information is

available at the end of the article

\begin{abstract}
In this paper, the change-point estimator for the shape parameter is proposed in a negative associated gamma random variable sequence. Suppose that $X_{1}, \ldots, X_{n}$ are negative associated random variables satisfying that $X_{1}, \ldots, X_{\left[n \tau_{0}\right]}$ are identically distributed with $\Gamma\left(x ; v_{1}, \lambda\right)$, and that $X_{\left[n \tau_{0}\right]+1}, \ldots, X_{n}$ are identically distributed with $\Gamma\left(x ; v_{2}, \lambda\right)$; the change point $\tau_{0}$ is unknown. The weak and strong consistency, and the weak and strong convergence rate of the change-point estimator, are given by the CUSUM method. Furthermore, the $O_{p}$ convergence rate of the change-point estimator is presented under the local alternative hypothesis condition.
\end{abstract}

MSC: $62 \mathrm{~F} 12 ; 62 \mathrm{G} 10$

Keywords: change point; $\Gamma$-distribution; negative associated; shape parameter; convergence rate

\section{Introduction}

The gamma distribution occurs frequently in a variety of applications, especially in reliability, in survival analysis and in modeling income distributions. The density of a gammadistributed random variable $X$ with a shape parameter $v$ and a scale parameter $\lambda$ is given by

$$
f(x ; \nu, \lambda)=\frac{\lambda^{\nu}}{\Gamma(v)} x^{\nu-1} e^{-\lambda x} I \quad(x>0),
$$

where $I(\cdot)$ is the indicator function, $\Gamma(\cdot)$ is a $\Gamma$ function with $\Gamma(p)=\int_{0}^{\infty} e^{-x} x^{p-1} d x$.

The family of gamma distributions includes the chi-squared distribution, exponential distribution and Erlang distribution. For example, the gamma distribution is an Erlang distribution with a positive integer $v$. When the shape parameter $v=1$, the gamma distribution is an exponential distribution with parameter $\lambda$; when $\lambda=\frac{1}{2}$, the gamma distribution is a chi-squared distribution, with $2 v$ degrees of freedom. The shape parameter is especially of interest in reliability theory because the gamma distribution is either a decreasing failure rate (DFR), a constant or an increasing failure rate (IFR) according to whether the shape parameter is negative, zero or positive. The shape parameter also plays an important role in renewal theory when modeling arrival times of events.

As for the gamma distribution parameter change-point problems, Kander and Zacks [1] proposed a statistic for testing a change in the one-parameter exponential family; Hsu [2] 
considered a change point for the scale parameter of gamma random variables, assuming that the shape parameter was constant; Diaz [3] posed the Bayesian test regarding the scale parameter change point for the independent gamma variables; Gupta and Ramanayake [4], Ramanayake and Gupta [5] discussed a linear trend change for the exponential distribution; Ramanayake [6] proposed some tests for detecting a change in the shape parameter of gamma distributions assuming that $\lambda$ is constant. The strong consistency and convergence rate of the change-point estimator have been investigated by applying the moving averages method (Tan et al. [7]), assuming that there is at most one change point.

Change-point analysis is widely used in fields such as quality control, economics and finance, biostatistics and so on (see Page [8]; Bai and Perron [9]; Braun et al. [10]; Chen et al. [11]). Change-point problems have also received considerable attention due to the wide variety of applications and recent developments in computational methods. There is a considerable body of literature on change-point analysis that assume that the random variables being considered are independent.

Let $X_{1}, X_{2}, \ldots, X_{n}$ be a negative associated sequence that satisfies the conditions that $X_{1}, \ldots, X_{\left[n \tau_{0}\right]}$ have the common distribution $\Gamma\left(x ; v_{1}, \lambda\right)$, and that $X_{\left[n \tau_{0}\right]+1}, \ldots, X_{n}$ have the common distribution $\Gamma\left(x ; v_{2}, \lambda\right)$, where $\tau_{0}$ is an unknown parameter called the change point; $v_{1}, v_{2}$ are the shape parameters before and after change, respectively. In this paper, we assume that the scale parameter does not change, but the shape parameter is susceptible to change at an unknown time $\left[n \tau_{0}\right]$ in the sequence. Noticing that $\lambda X \sim \Gamma(x ; v, 1)$ and its distribution is not related to the scale parameter, logarithm transformations may be made for $\left\{X_{i}, i=1, \ldots, n\right\}$ as follows. Let

$$
Y_{i}=\ln \lambda X_{i}, \quad i=1,2, \ldots, n .
$$

It can be shown that the mean of $Y_{1}$ is $\mu_{1}=E Y_{1}=\Psi\left(v_{1}\right)$ and the mean of $Y_{\left[n \tau_{0}\right]+1}$ is $\mu_{2}=$ $E Y_{\left[n \tau_{0}\right]+1}=\Psi\left(v_{2}\right)$, where $\Psi(v)$ is the derivation of $\ln \Gamma(v)$; that is,

$$
\Psi(v)=\frac{d[\ln (\Gamma(v))]}{d v}=\frac{\Gamma^{\prime}(v)}{\Gamma(v)} .
$$

$\Psi(v)$ can be expressed, as in [12, p.16], by

$$
\Psi(v)=-\gamma+\int_{0}^{+\infty} \frac{e^{-t}-e^{-v t}}{1-e^{-t}} d t,
$$

where $\gamma$ is the Euler-Mascheroni constant, that is, $\gamma=-\int_{0}^{+\infty} e^{-x} \ln x d x$. Since $\Psi^{\prime}(v)=$ $\int_{0}^{+\infty} \frac{t}{1-e^{-t}} e^{-v t} d t>0$, hence $\Psi(v)$ is an increasing function in $(0,+\infty)$.

Define

$$
\begin{aligned}
& U_{k}=\sum_{i=1}^{k} Y_{i}-\frac{k}{n} \sum_{i=1}^{n} Y_{i}, \\
& \rho_{0}=v_{1}-v_{2}, \quad \mu_{0}=\mu_{1}-\mu_{2} .
\end{aligned}
$$

Since

$$
U_{k}=\sum_{i=1}^{k} \ln \lambda X_{i}-\frac{k}{n} \sum_{i=1}^{n} \ln \lambda X_{i}=\sum_{i=1}^{k} \ln X_{i}-\frac{k}{n} \sum_{i=1}^{n} \ln X_{i},
$$


are not related to the scale parameter $\lambda$, then if we know in advance or by test that there is a change in the shape parameter, we may define the estimator of the change point $\tau_{0}$ as

$$
\widehat{\tau}=\frac{1}{n} \min \left\{k:\left|U_{k}\right|=\max _{1 \leq j \leq n}\left|U_{j}\right|\right\} .
$$

For convenience, throughout this paper, $c, c_{1}, \ldots$ represent a constant which is independent of $n$ and may take different values in different expressions.

The paper is arranged as follows. In Section 2, the change-point estimator $\widehat{\tau}$ is proposed based on the CUSUM method by an appropriate logarithm transformation for $\left\{X_{i}, i=\right.$ $1, \ldots, n\}$, and its constancy and convergence rate are investigated. The proofs of theorems are given in Section 3.

\section{Main results}

Theorem 1 Assume that $X_{1}, X_{2}, \ldots, X_{n}$ is a negative associated random variable sequence satisfying the conditions that $X_{1}, \ldots, X_{\left[n \tau_{0}\right]}$ are identically distributed with $\Gamma\left(x ; v_{1}, \lambda\right)$, and $X_{\left[n \tau_{0}\right]+1}, \ldots, X_{n}$ are identically distributed with $\Gamma\left(x ; v_{2}, \lambda\right)$. Let

$$
k_{0}=\left[n \tau_{0}\right], \quad \widehat{k}=[n \widehat{\tau}], \quad k=[n \tau] \text { for some } 0<\tau<1,
$$

where $[A]$ denotes the integer part of a number $A$. If the $\rho_{0}=v_{1}-v_{2}$ is a non-zero constant, then $\widehat{\tau}$ is a consistent estimator of $\tau_{0}$ and

$$
\left|\widehat{\tau}-\tau_{0}\right|=o_{P}\left(n^{-\frac{1}{2}} l(n)\right)
$$

where $l(n)$ is a slowly varying function with $\lim _{n \rightarrow \infty} l(n)=+\infty$.

Theorem 2 Assume that the conditions of Theorem 1 hold, then $\widehat{\tau}$ is a strong consistent estimator of $\tau_{0}$, and

$$
\left|\widehat{\tau}-\tau_{0}\right|=o\left(n^{-\frac{1}{2}+\delta}\right), \quad \text { a.s. for some } 0<\delta<\frac{1}{2} .
$$

Next, we will study the $O_{P}$ convergence rate of $\widehat{\tau}$ under the local alternative hypothesis; that is, $\rho_{0}$ is not a constant independent of $n$, but it depends on $n$ and is denoted by $\rho_{n}$. Noticing that if $\rho_{n}$ is large, the change-point estimation is usually quite precise. In practice it may be more important to construct confidence intervals for $\tau_{0}$ when $\rho_{n}$ is small. We hence assume that $\rho_{n} \longrightarrow 0$ as $n \longrightarrow \infty$. It can be seen that the results obtained in the above theorems cannot be applied here, and we need to establish stronger results than those obtained in the above theorems.

Notice that $\mu_{i}=\Psi\left(v_{i}\right), i=1,2$. Then, by the mean theorem, $\mu_{n}$ (under the local alternative hypothesis, denoting $\mu_{0}$ as $\mu_{n}$ ) can be expressed as

$$
\mu_{n}=\mu_{1}-\mu_{2}=\Psi^{\prime}(\tilde{v})\left(v_{1}-v_{2}\right)=\Psi^{\prime}(\tilde{v}) \rho_{n},
$$

where $\tilde{v}$ lies between $v_{1}$ and $v_{2}$. Hence, with some added conditions, $\mu_{n}$ is equal to $\rho_{n}$ in practice. 
Theorem 3 Assume that $X_{1}, X_{2}, \ldots, X_{n}$ is a negative associated random variable sequence, and $X_{1}, \ldots, X_{\left[n \tau_{0}\right]}$ are identically distributed by $\Gamma\left(x ; v_{1}, \lambda\right)$, and $X_{\left[n \tau_{0}\right]+1}, \ldots, X_{n}$ are identically distributed by $\Gamma\left(x ; \nu_{2}, \lambda\right)$. If $\mu_{n}$ satisfies

$$
\mu_{n} \rightarrow 0, \quad \sqrt{n} \mu_{n} \rightarrow \infty
$$

then

$$
\left|\widehat{\tau}-\tau_{0}\right|=O_{P}\left(\frac{1}{n \mu_{n}^{2}}\right) .
$$

Remark 1 Theorems 1 and 2 give the weak and strong consistency and convergence rates for the change-point estimator $\widehat{\tau}$ of the shape parameter in a gamma distribution. In Theorem 3, the $O_{P}$ convergence rate of the change-point estimator $\widehat{\tau}$ of the shape parameter is proposed under the local alternative condition, and it is one of the necessary conditions for studying the limiting distribution of $\widehat{\tau}$. Having this $O_{P}$ value, we can study the limiting distribution of $\widehat{\tau}$. This will be the subject of a future paper.

\section{Proof of the theorem}

To prove the above theorems, we first consider the following lemmas.

Lemma 1 Let $A_{1}, A_{2}, \ldots, A_{m}$ be disjoint subsets of $\{1,2, \ldots, n\}$, and let $a_{i}=\sharp\left(A_{i}\right)$ be the number of elements in $A_{i}, i=1,2, \ldots, m$. Assume that $Z_{1}, Z_{2}, \ldots, Z_{n}$ are negative associated variables, then if

$$
f_{i}: R^{a_{i}} \rightarrow R, \quad i=1,2, \ldots, m
$$

are the increasing (or decreasing) positive functions for every element, then $f_{1}\left(Z_{j}, j \in\right.$ $\left.A_{1}\right), \ldots, f_{m}\left(Z_{j}, j \in A_{m}\right)$ are the negative associated variables.

Proof See Joag-Dev and Proschan [13].

Lemma 2 Let $\left\{Z_{j}, j \in N\right\}$ be a negative associated sequence with zero mean satisfying $\beta_{p}=\sup _{j \in N} E\left|Z_{j}\right|^{p}<\infty$ for some $p \geq 2$. Denoting $S_{a, k}=\sum_{j=0}^{k-1} Z_{a+j}$, then there exist constants $C_{p}, K_{p} \geq 1$ related to $p$, for all $a, n \in N$, such that

$$
E\left|S_{1, n}\right|^{p} \leq C_{p} n^{\frac{p}{2}-1} \sum_{j=1}^{n} E\left|Z_{j}\right|^{p} ; \quad E\left(\max _{1 \leq k \leq n}\left|S_{a, k}\right|\right)^{p} \leq K_{p} \beta_{p} n^{\frac{p}{2}} .
$$

Proof See Su, Zhao and Wang [14].

Lemma 3 Let $\left\{Z_{n}, n \geq 1\right\}$ be a negative associated sequence, if $\left\{b_{k}, k \geq 1\right\}$ is an increasing number serial, then $\forall \varepsilon>0$ and $m \leq n$, we have

$$
\begin{aligned}
& P\left(\max _{1 \leq k \leq n}\left|\frac{1}{b_{k}} \sum_{i=1}^{k}\left(X_{i}-E X_{i}\right)\right| \geq \varepsilon\right) \leq \frac{8}{\varepsilon^{2}} \sum_{j=1}^{n} \frac{\operatorname{Var}\left(X_{j}\right)}{b_{j}^{2}} ; \\
& P\left(\max _{m \leq k \leq n}\left|\frac{1}{b_{k}} \sum_{i=1}^{k}\left(X_{i}-E X_{i}\right)\right| \geq \varepsilon\right) \leq \frac{4}{\varepsilon^{2}}\left(\sum_{j=1}^{m} \frac{\operatorname{Var}\left(X_{j}\right)}{b_{m}^{2}}+8 \sum_{j=m+1}^{n} \frac{\operatorname{Var}\left(X_{j}\right)}{b_{j}^{2}}\right) .
\end{aligned}
$$


Proof See Hu and Zhang [15].

Proof of Theorem 1 Noticing that $\ln (\lambda x)$ is an increasing positive function and $X_{1}, \ldots, X_{n}$ are negative associated sequences, we have from Lemma 1 that $\left\{Y_{i}, i=1, \ldots, n\right\}$ are negative associated sequences. Without loss of generality, assuming that $v_{1}>v_{2}$, by the increasing character of $\Psi(v)$ in $(0, \infty)$, we know that $\mu_{0}=\mu_{1}-\mu_{2}>0$. By simple computation, it can be shown that

$$
E U_{k_{0}}=\frac{k_{0}\left(n-k_{0}\right)}{n}\left(\mu_{1}-\mu_{2}\right)=n \tau_{0}\left(1-\tau_{0}\right) \mu_{0},
$$

and

$$
E U_{k}=\left\{\begin{array}{ll}
\frac{k\left(n-k_{0}\right)}{n} \mu_{0}, & k \leq k_{0}, \\
\frac{(n-k) k_{0}}{n} \mu_{0}, & k>k_{0},
\end{array}= \begin{cases}n \tau\left(1-\tau_{0}\right) \mu_{0}, & k \leq k_{0}, \\
n(1-\tau) \tau_{0} \mu_{0}, & k>k_{0} .\end{cases}\right.
$$

Hence,

$$
\begin{aligned}
\left|E U_{k_{0}}\right|-\left|E U_{k}\right| & = \begin{cases}n\left(1-\tau_{0}\right)\left(\tau_{0}-\tau\right) \mu_{0}, & k \leq k_{0}, \\
n \tau_{0}\left(\tau-\tau_{0}\right) \mu_{0}, & k>k_{0},\end{cases} \\
& \geq n \tau^{*} \mu_{0}\left|\tau-\tau_{0}\right|,
\end{aligned}
$$

where $\tau^{*}=\min \left\{\tau_{0}, 1-\tau_{0}\right\}$.

From the triangle inequality, it can easily be shown that

$$
\left|U_{k}\right|-\left|U_{k_{0}}\right| \leq 2 \max _{1 \leq k \leq n}\left|U_{k}-E U_{k}\right|+\left|E U_{k}\right|-\left|E U_{k_{0}}\right|
$$

namely,

$$
\left|E U_{k_{0}}\right|-\left|E U_{k}\right| \leq 2 \max _{1 \leq k \leq n}\left|U_{k}-E \mu_{k}\right|+\left|U_{k_{0}}\right|-\left|U_{k}\right|
$$

Noticing that $\left|U_{k_{0}}\right| \leq\left|U_{\hat{k}}\right|$, hence we have

$$
\left|E U_{k_{0}}\right|-\left|E U_{\widehat{k}}\right| \leq 2 \max _{1 \leq k \leq n}\left|U_{k}-E U_{k}\right|
$$

Let $Y_{i}^{*}=Y_{i}-E Y_{i}$, then by (16) and (17), it follows that

$$
\begin{aligned}
n \tau^{*} \mu_{0}\left|\tau-\tau_{0}\right| & \leq 2 \max _{1 \leq k \leq n}\left|U_{k}-E U_{k}\right|=2 \max _{1 \leq k \leq n}\left|\sum_{i=1}^{k} Y_{i}^{*}-\frac{k}{n} \sum_{i=1}^{n} Y_{i}^{*}\right| \\
& =2 \max _{1 \leq k \leq n}\left|\frac{n-k}{n} \sum_{i=1}^{k} Y_{i}^{*}-\frac{k}{n} \sum_{i=k+1}^{n} Y_{i}^{*}\right| \\
& \leq 2 \max _{1 \leq k \leq n}\left|\sum_{i=1}^{k} Y_{i}^{*}\right|+2 \max _{1 \leq k \leq n}\left|\sum_{i=k+1}^{n} Y_{i}^{*}\right| .
\end{aligned}
$$


Hence,

$$
\begin{aligned}
P\left(g_{1}(n)\left|\widehat{\tau}-\tau_{0}\right|>\varepsilon\right) & =P\left(\left|\widehat{\tau}-\tau_{0}\right|>\frac{\varepsilon}{g_{1}(n)}\right) \\
& \leq P\left(\frac{2}{n \tau^{*} \mu_{0}}\left\{\max _{1 \leq k \leq n}\left|\sum_{i=1}^{k} Y_{i}^{*}\right|+\max _{1 \leq k \leq n}\left|\sum_{i=k+1}^{n} Y_{i}^{*}\right|\right\}>\frac{\varepsilon}{g_{1}(n)}\right) \\
& \leq P\left(\max _{1 \leq k \leq n}\left|\sum_{i=1}^{k} Y_{i}^{*}\right|>\frac{n \tau^{*} \mu_{0}}{4 g_{1}(n)} \varepsilon\right)+P\left(\max _{1 \leq k \leq n}\left|\sum_{i=k+1}^{n} Y_{i}^{*}\right|>\frac{n \tau^{*} \mu_{0}}{4 g_{1}(n)} \varepsilon\right) \\
& \widehat{=} A_{1}+A_{2} .
\end{aligned}
$$

Since $Y_{1}, Y_{2}, \ldots, Y_{n}$ are the negative associated variables, by the Markov inequality and Lemma $2, \forall r>2$, we have

$$
\begin{aligned}
A_{1} & \leq E\left(\max _{1 \leq k \leq n}\left|\sum_{i=1}^{k} Y_{i}^{*}\right|\right)^{r} /\left(\frac{n \tau^{*} \mu_{0} \varepsilon}{4 g_{1}(n)}\right)^{r}=\frac{4^{r}}{\left(\varepsilon \tau^{*} \mu_{0}\right)^{r}} \frac{g_{1}^{r}(n)}{n^{r}} E\left(\max _{1 \leq k \leq n}\left|\sum_{i=1}^{k} Y_{i}^{*}\right|\right)^{r} \\
& \leq \frac{4^{r}}{\left(\varepsilon \tau^{*} \mu_{0}\right)^{r}} \frac{g_{1}^{r}(n)}{n^{r}} K_{r} \beta_{r} n^{\frac{r}{2}} \leq \frac{4^{r} K_{r} \beta_{r}}{\left(\varepsilon \tau^{*} \mu_{0}\right)^{r}}\left(\frac{n^{\frac{1}{2}}}{g_{1}(n)}\right)^{-r},
\end{aligned}
$$

where $\beta_{r}=\max \left\{E\left|Y_{1}^{*}\right|^{r}, E\left|Y_{\left[n \tau_{0}\right]+1}^{*}\right|^{r}\right\}$ is a constant independent of $n$. Similar arguments give that

$$
A_{2} \leq \frac{4^{r} K_{r} \beta_{r}}{\left(\varepsilon \tau^{*} \mu_{0}\right)^{r}}\left(\frac{n^{\frac{1}{2}}}{g_{1}(n)}\right)^{-r} .
$$

Hence, if we choose $g_{1}(n)=n^{\frac{1}{2}} l^{-1}(n)$, where $l(n)$ is a slowly varying function satisfying $\lim _{n \rightarrow \infty} l(n)=+\infty$, then combining (19)-(21) we have, as $n \rightarrow \infty$,

$$
P\left(g_{1}(n)\left|\widehat{\tau}-\tau_{0}\right|>\varepsilon\right) \longrightarrow 0
$$

that is, $\widehat{\tau}$ is the weak consistent estimator of $\tau_{0}$, and

$$
\left|\widehat{\tau}-\tau_{0}\right|=o_{P}\left(n^{-\frac{1}{2}} l(n)\right) \text {. }
$$

Proof of Theorem 2 From (19) to (21) we have, for $\forall \varepsilon>0$,

$$
\sum_{n=1}^{\infty} P\left(g_{2}(n)\left|\widehat{\tau}-\tau_{0}\right|>\varepsilon\right) \leq \sum_{n=1}^{\infty} \frac{4^{r} K_{r} \beta_{r}}{\left(\varepsilon \tau^{*} \mu_{0}\right)^{r}}\left(\frac{n^{\frac{1}{2}}}{g_{2}(n)}\right)^{-r}
$$

if we choose $g_{2}(n)=n^{\frac{1}{2}-\delta}$ for some $0<\delta<\frac{1}{2}$. Let $r>\frac{1}{\delta}$, then we have, as $n \rightarrow \infty$,

$$
\sum_{n=1}^{\infty} P\left(g_{2}(n)\left|\widehat{\tau}-\tau_{0}\right|>\varepsilon\right)<\infty
$$

By the Borel-Cantelli lemma, we obtain that $\widehat{\tau}$ is the strongly consistent estimator of $\tau$, and

$$
\left|\widehat{\tau}-\tau_{0}\right|=o\left(n^{-\frac{1}{2}+\delta}\right) \quad \text { a.s. for some } 0<\delta<\frac{1}{2}
$$


Proof of Theorem 3 To this end, we choose a value $0<\theta<\frac{1}{2}$ such that $\tau \in(\theta, 1-\theta)$. By (8) and (19)-(21) $\left(g_{1}(n)=1\right)$, it is easily found that $\widehat{\tau}$ is a consistent estimator of $\tau_{0}$. Therefore, for every $\varepsilon>0, P(\widehat{\tau} \notin(\theta, 1-\theta))<\varepsilon$. Thus, we now have only to examine the behavior of $U_{k}$ over those $k$ for which $n \theta \leq k \leq n(1-\theta)$. To prove $\left|\widehat{\tau}-\tau_{0}\right|=O_{P}\left(\frac{1}{n \mu_{n}^{2}}\right)$, we shall prove that

$$
P\left(\left|\widehat{\tau}-\tau_{0}\right|>\frac{M}{n \mu_{0}^{2}}\right) \rightarrow 0
$$

when $M \longrightarrow \infty$. For every $M>0$, define

$$
D_{n, M}=\left\{k: n \theta \leq k \leq n(1-\theta),\left|k-k_{0}\right|>\frac{M}{\mu_{n}^{2}}\right\} .
$$

Then we have

$$
\begin{aligned}
P\left(\left|\widehat{\tau}-\tau_{0}\right|>\frac{M}{n \mu_{0}^{2}}\right) & \leq P(\widehat{\tau} \notin(\theta, 1-\theta))+P\left(\left|\widehat{\tau}-\tau_{0}\right|>\frac{M}{n \mu_{n}^{2}}, \hat{\tau} \in(\theta, 1-\theta)\right) \\
& \leq \varepsilon+P\left(\sup _{k \in D_{n, M}}\left|U_{k}\right| \geq\left|U_{k_{0}}\right|\right) .
\end{aligned}
$$

Since

$$
\begin{aligned}
& P\left(\sup _{k \in D_{n, M}}\left|U_{k}\right| \geq\left|U_{k_{0}}\right|\right) \\
& \quad=P\left(\sup _{k \in D_{n, M}}\left|U_{k}\right| \geq U_{k_{0}}, U_{k_{0}} \geq 0\right)+P\left(\sup _{k \in D_{n, M}}\left|U_{k}\right| \geq-U_{k_{0}}, U_{k_{0}}<0\right) \\
& \quad=P\left(\sup _{k \in D_{n, M}}\left|U_{k}\right|-U_{k_{0}} \geq 0, U_{k_{0}} \geq 0\right)+P\left(\sup _{k \in D_{n, M}}\left|U_{k}\right|+U_{k_{0}} \geq 0, U_{k_{0}}<0\right) \\
& \quad \leq P\left(\sup _{k \in D_{n, M}}\left(U_{k}-U_{k_{0}}\right) \geq 0\right)+P\left(\inf _{k \in D_{n, M}}\left(U_{k}+U_{k_{0}}\right) \leq 0\right) \\
& \quad \widehat{=} B_{1}+B_{2} .
\end{aligned}
$$

Noticing that $U_{k}+U_{k_{0}} \leq 0$ implies $U_{k}-E U_{k}+U_{k_{0}}-E U_{k_{0}} \leq-E U_{k}-E U_{k_{0}} \leq-E U_{k_{0}}$, which in turn implies that

$$
U_{k}-E U_{k} \leq-\frac{1}{2} E U_{k_{0}} \quad \text { or } \quad U_{k_{0}}-E U_{k_{0}} \leq-\frac{1}{2} E U_{k_{0}} .
$$

Since $E U_{k_{0}}>0$, we have

$$
\left|U_{k}-E U_{k}\right| \geq \frac{1}{2} E U_{k_{0}} \quad \text { or } \quad\left|U_{k_{0}}-E U_{k_{0}}\right| \geq \frac{1}{2} E U_{k_{0}} .
$$

Furthermore, we obtain

$$
\begin{aligned}
B_{2} & \leq P\left(\sup _{k \in D_{n, M}}\left|U_{k}-E U_{k}\right| \geq \frac{1}{2} E U_{k_{0}}\right)+P\left(\left|U_{k_{0}}-E U_{k_{0}}\right| \geq \frac{1}{2} E U_{k_{0}}\right) \\
& \widehat{=} D_{1}+D_{2} .
\end{aligned}
$$


It can be seen from the definition of $D_{n, M}$ that

$$
\begin{aligned}
D_{1} & \leq P\left(\sup _{n \theta \leq k \leq n(1-\theta)}\left|U_{k}-E U_{k}\right| \geq \frac{1}{2} E U_{k_{0}}\right) \\
& =P\left(\sup _{n \theta \leq k \leq n(1-\theta)}\left|\sum_{i=1}^{k} Y^{*}-\frac{k}{n} \sum_{i=1}^{n} Y^{*}\right| \geq \frac{1}{2} n \tau_{0}\left(1-\tau_{0}\right) \mu_{n}\right) \\
& \leq P\left(\max _{1 \leq k \leq n}\left|\sum_{i=1}^{k} Y_{i}^{*}-\frac{k}{n} \sum_{i=1}^{n} Y_{i}^{*}\right| \geq \frac{1}{2} n \tau_{0}\left(1-\tau_{0}\right) \mu_{n}\right) \\
& \leq P\left(\max _{1 \leq k \leq n}\left|\sum_{i=1}^{k} Y_{i}^{*}\right| \geq \frac{1}{4} n \tau_{0}\left(1-\tau_{0}\right) \mu_{n}\right)+P\left(\max _{1 \leq k \leq n}\left|\frac{k}{n} \sum_{i=1}^{n} Y_{i}^{*}\right| \geq \frac{1}{4} n \tau_{0}\left(1-\tau_{0}\right) \mu_{n}\right) \\
& \leq P\left(\max _{1 \leq k \leq n}\left|\sum_{i=1}^{k} Y_{i}^{*}\right| \geq \frac{1}{4} n \tau_{0}\left(1-\tau_{0}\right) \mu_{n}\right)+P\left(\left|\sum_{i=1}^{n} Y_{i}^{*}\right| \geq \frac{1}{4} n \tau_{0}\left(1-\tau_{0}\right) \mu_{n}\right) \\
& \widehat{\equiv} E_{1}+E_{2} .
\end{aligned}
$$

Because $Y_{1}^{*}, \ldots, Y_{n}^{*}$ are negative associated variables, by the Markov inequality, Lemma 2 and (8), $\forall p \geq 2$, we obtain

$$
\begin{aligned}
E_{1} & =P\left(\max _{1 \leq k \leq n}\left|\sum_{i=1}^{k} Y_{i}^{*}\right| \geq \frac{1}{4} n \tau_{0}\left(1-\tau_{0}\right) \mu_{n}\right) \\
& \leq E\left(\max _{k}\left|\sum_{i=1}^{k} Y_{i}^{*}\right|^{p}\right) /\left(\frac{1}{4} n \tau_{0}\left(1-\tau_{0}\right) \mu_{n}\right)^{p} \\
& \leq \frac{4^{p}}{\left[n \tau_{0}\left(1-\tau_{0}\right) \mu_{n}\right]^{p}} K_{p} \beta_{p} n^{\frac{p}{2}} \leq c_{1} \frac{1}{\left(n^{\frac{1}{2}} \mu_{n}\right)^{p}} \longrightarrow 0 \quad\left(\text { as } n \mu_{n}^{2} \longrightarrow \infty\right) .
\end{aligned}
$$

Similar arguments give

$$
\begin{aligned}
E_{2} & =P\left(\left|\sum_{i=1}^{n} Y_{i}^{*}\right| \geq \frac{1}{4} n \tau_{0}\left(1-\tau_{0}\right) \mu_{n}\right) \\
& \leq \frac{4^{p}}{\left[n \tau_{0}\left(1-\tau_{0}\right) \mu_{n}\right]^{p}} C_{p} n^{\frac{p}{2}-1} \sum_{i=1}^{n} E\left|Y^{*}\right|^{p} \leq c_{2} \frac{1}{\left(n^{\frac{1}{2}} \mu_{n}\right)^{p}} \longrightarrow 0 \quad\left(\text { as } n \mu_{n}^{2} \longrightarrow \infty\right) .
\end{aligned}
$$

Combining (28)-(30), we see that

$$
D_{1} \longrightarrow 0 \quad \text { as } n \mu_{n}^{2} \rightarrow \infty
$$

Similar arguments as those for $D_{1}$ give

$$
\begin{aligned}
D_{2} & =P\left(\left|\sum_{i=1}^{k_{0}} Y_{i}^{*}-\frac{k_{0}}{n} \sum_{i=1}^{n} Y_{i}^{*}\right| \geq \frac{1}{2} n \tau_{0}\left(1-\tau_{0}\right) \mu_{n}\right) \\
& \leq P\left(\left|\sum_{i=1}^{k_{0}} Y_{i}^{*}\right| \geq \frac{1}{4} n \tau_{0}\left(1-\tau_{0}\right) \mu_{n}\right)
\end{aligned}
$$




$$
\begin{aligned}
& +P\left(\left|\sum_{i=1}^{n} Y_{i}^{*}\right| \geq \frac{1}{4} n \tau_{0}\left(1-\tau_{0}\right) \mu_{n}\right) \\
\leq & c_{3} \frac{1}{\left(n^{\frac{1}{2}} \mu_{n}\right)^{p}} \longrightarrow 0 \quad \text { as } n \mu_{n}^{2} \longrightarrow \infty .
\end{aligned}
$$

Combining (27), (31) and (32), we obtain

$$
B_{2} \longrightarrow 0 \text {. }
$$

Now consider $B_{1}$. Because of symmetry, we only consider the case of $k \leq k_{0}$. The event $U_{k}-U_{k_{0}} \geq 0$ implies that

$$
\begin{aligned}
U_{k}- & E U_{k}-\left(U_{k_{0}}-E U_{k_{0}}\right) \\
\geq & E U_{k_{0}}-E U_{k} \\
& \Longleftrightarrow\left(\sum_{i=1}^{k} Y_{i}^{*}-\frac{k}{n} \sum_{i=1}^{n} Y_{i}^{*}\right)-\left(\sum_{i=1}^{k_{0}} Y_{i}^{*}-\frac{k_{0}}{n} \sum_{i=1}^{n} Y_{i}^{*}\right) \geq n \tau^{*} \mu_{n}\left|\tau-\tau_{0}\right| \\
& \Longleftrightarrow\left(-\sum_{i=k+1}^{k_{0}} Y_{i}^{*}-\frac{k-k_{0}}{n} \sum_{i=1}^{n} Y_{i}^{*}\right) \geq n \tau^{*} \mu_{n}\left|\tau-\tau_{0}\right| \\
& \Longleftrightarrow \frac{-1}{k_{0}-k} \sum_{i=k+1}^{k_{0}} Y_{i}^{*}+\frac{1}{n} \sum_{i=1}^{n} Y_{i}^{*} \geq \tau^{*} \mu_{n} .
\end{aligned}
$$

Therefore,

$$
\begin{aligned}
B_{1} & \leq P\left(\max _{k \in D_{n, M}}\left|\frac{1}{k_{0}-k} \sum_{i=k+1}^{k_{0}} Y_{i}^{*}+\frac{1}{n} \sum_{i=1}^{n} Y_{i}^{*}\right| \geq \tau^{*} \mu_{n}\right) \\
& \leq P\left(\max _{k \in D_{n, M}}\left|\frac{1}{k_{0}-k} \sum_{i=k+1}^{k_{0}} Y_{i}^{*}\right| \geq \frac{1}{2} \tau^{*} \mu_{n}\right)+P\left(\max _{k \in D_{n, M}}\left|\sum_{i=1}^{n} Y_{i}^{*}\right| \geq \frac{1}{2} n \tau^{*} \mu_{n}\right) \\
& \widehat{=} F_{1}+F_{2} .
\end{aligned}
$$

From the Markov inequality, Lemma 2 and (8), we obtain

$$
F_{2} \leq 2^{p} \frac{C_{P}}{\left(n \tau^{*} \mu_{n}\right)^{p}} n^{\frac{p}{2}} \leq c_{4}\left(\frac{1}{n \mu_{n}^{2}}\right)^{\frac{p}{2}} \rightarrow 0 .
$$

Denoting $\sigma_{1}^{2}=\operatorname{Var}\left(Y_{1}^{*}\right)$, from Lemma 3 and (8), we obtain

$$
\begin{aligned}
F_{1} & =P\left(\max _{n \theta \leq k \leq k_{0}-\frac{M}{\mu_{n}^{2}}}\left|\frac{1}{k_{0}-k} \sum_{i=k+1}^{k_{0}} Y_{i}^{*}\right| \geq \frac{1}{2} \tau^{*} \mu_{n}\right) \\
& =P\left(\max _{\frac{M}{\mu_{n}^{2}} \leq k_{0}-k \leq k_{0}-n \theta}\left|\frac{1}{k_{0}-k} \sum_{j=1}^{k_{0}-k} Y_{k_{0}+1-j}^{*}\right| \geq \frac{1}{2} \tau^{*} \mu_{n}\right) \\
& =P\left(\max _{\frac{M}{\mu_{n}^{2}} \leq t \leq k_{0}-n \theta}\left|\frac{1}{t} \sum_{j=1}^{t} Y_{k_{0}+1-j}^{*}\right| \geq \frac{1}{2} \tau^{*} \mu_{n}\right)
\end{aligned}
$$




$$
\begin{aligned}
& \leq \frac{4 * 4}{\left(\tau^{*} \mu_{n}\right)^{2}}\left\{\sum_{j=1}^{\frac{M}{\mu_{n}^{2}}} \frac{\operatorname{Var}\left(Y_{k_{0}+1-j}^{*}\right)}{\left(\frac{M}{\mu_{n}^{2}}\right)^{2}}+8 \sum_{j=\frac{M}{\mu_{n}^{2}}+1}^{k_{0}-n \theta} \frac{\operatorname{Var}\left(Y_{k_{0}+1-j}^{*}\right)}{j^{2}}\right\} \\
& \leq 16 \frac{\sigma_{1}^{2}}{\left(\tau^{*}\right)^{2} \mu_{n}^{2}}\left\{\frac{\mu_{n}^{2}}{M}+8 \sum_{j=\frac{M}{\mu_{n}^{2}+1}}^{k_{0}-n \theta} \frac{1}{j(j-1)}\right\} \\
& =16 \frac{\sigma_{1}^{2}}{\left(\tau^{*}\right)^{2} \mu_{n}^{2}}\left\{\frac{\mu_{n}^{2}}{M}+8\left(\frac{\mu_{n}^{2}}{M}-\frac{1}{k_{0}-n \theta-1}\right)\right\} \\
& =16 \frac{\sigma_{1}^{2}}{\left(\tau^{*}\right)^{2}}\left(\frac{9}{M}-\frac{8}{\left(k_{0}-n \theta-1\right) \mu_{n}^{2}}\right) \rightarrow 0 \quad\left(\text { as } n \mu_{n}^{2} \rightarrow \infty, M \rightarrow \infty\right) .
\end{aligned}
$$

Combining (34)-(36), we have

$$
B_{1} \longrightarrow 0 .
$$

From (24), (33) and (37), we know that (23) holds; that is, Theorem 3 is proved.

\section{Competing interests}

The authors declare that they have no competing interests.

\section{Authors' contributions}

C-cT conceived of the study questions which can be done, participated in the proofs and drafted the manuscript. B-qM participated in the proofs and provided the related reference. X-cZ participated in the proof of Theorems and helped to draft the manuscript. All authors read and approved the final manuscript.

\section{Author details}

${ }^{1}$ School of Mathematics, Hefei University of Technology, Heifei, Anhui 230009, China. ${ }^{2}$ Department of Statistics and Finance, University of Science and Technology of China, Hefei, Anhui 230026, China. ${ }^{3}$ Department of Mathematics and Computer Science, Tongling University, Tongling, Anhui 244000, China.

\section{Acknowledgements}

We are grateful to the three referees for the valuable comments and advices that led to substantial improvement of an original draft of this paper.

The work of Dr. Tan was partially supported by a grant from the National Natural Science Foundation of China (No. 11201108), the Humanities and Social Sciences Project from Ministry of Education of China (No. 12YJC910007, 11 YJC790311), the Anhui Provincial Natural Science Foundation (No. 1208085QA12) and the twelfth Five-Year plan National Science and Technology major Project (2012ZX10004609).

\section{Received: 25 October 2012 Accepted: 18 March 2013 Published: 8 April 2013}

\section{References}

1. Kander, Z, Zacks, S: Test procedures for possible changes in parameters of statistical distributions occurring at unknown time points. Ann. Math. Stat. 37, 1196-1210 (1966)

2. Hsu, DA: Detecting shifts of parameter in gamma sequences with applications to stock price and air traffic flow analysis. J. Am. Stat. Assoc. 74, 31-40 (1979)

3. Diaz, J: Bayesian detection of a change of scale parameter in sequences of independent gamma random variables. J. Econom. 19, 23-29 (1982)

4. Ramanayake, A, Gupta, AK: Change points with linear trend followed by abrupt change for the exponential distribution. J. Stat. Comput. Simul. 72(4), 263-278 (2002)

5. Gupta, AK, Ramanayake, A: Change points with linear trend for the exponential distribution. J. Stat. Plan. Inference 93 , 181-195 (2001)

6. Ramanayake, A: Tests for a change point in the shape parameter of Gamma random variables. Commun. Stat., Theory Methods 33(4), 821-833 (2004)

7. Tan, CC, Zhao, LC, Miao, BQ: Inference and application in finance of $\Gamma$-distribution with at most one change-point. J. Syst. Sci. Math. Sci. 27, 2-10 (2007) (in Chinese)

8. Page, ES: Continuous inspection schemes. Biometrika 42, 100-114 (1954)

9. Bai, J, Perron, P: Estimating and testing linear models with multiple structural changes. Econometrica 66, 47-78 (1998)

10. Braun, JV, et al.: Multiple changepoint fitting via quasilikelihood with application to DNA sequence segmentation. Biometrika 87(2), 301-314 (2000) 
11. Chen, $C$, et al.: Nonparametric estimation of structural changepoints in volatility models for time series. J. Econom 126, 79-114 (2005)

12. Magnus, W, Oberhettinger, F, Soni, RP: Formulas and Theorems for the Special Functions of Mathematical Physics Springer, Berlin (1966)

13. Joag-Dev, K, Proschan, F: Negative association of random variables with application. Ann. Stat. 11, 286-295 (1983)

14. Su, C, Zhao, LC, Wang, YB: Moment inequalities and weak convergence for negatively associated sequences. Sci. China Ser. A 40(2), 172-182 (1997)

15. Hu, SH, Zhang, LS: Hajek-Renyi-type inequality under second moment conditions and its application. Acta Math. Appl. Sin. 28(2), 227-235 (2005) (in Chinese)

doi:10.1186/1029-242X-2013-161

Cite this article as: Tan et al.: Statistical inference for the shape parameter change-point estimator in negative associated gamma distribution. Journal of Inequalities and Applications 2013 2013:161.

Submit your manuscript to a SpringerOpen ${ }^{\circ}$ journal and benefit from:

- Convenient online submission

- Rigorous peer review

- Immediate publication on acceptance

- Open access: articles freely available online

- High visibility within the field

- Retaining the copyright to your article

Submit your next manuscript at $>$ springeropen.com 